\title{
Influence of copolymer composition on the transport properties of conducting copolymers: poly(aniline-co-o-anisidine)
}

\author{
S S UMARE*, A D BORKAR ${ }^{\dagger}$ and M C GUPTA ${ }^{\dagger}$ \\ Department of Chemistry, Visvesvaraya Regional College of Engineering, Nagpur 440 011, India \\ ${ }^{\dagger}$ Department of Chemistry, Nagpur University, Nagpur 440 010, India
}

MS received 16 August 2000; revised 19 March 2002

\begin{abstract}
The effect of different compositions of monomers on the transport properties of copolymers by various techniques such as optical, electrical and magnetic has been investigated and compared with the homopolymers. The UV-visible absorption spectra show a hypsochromic shift with an increase in the o-anisidine content in copolymers indicating a decrease in the extent for conjugation (i.e. an increase in the bandgap). From temperature dependence of electrical conductivity the transport parameters such as charge localization length and average hopping distance are calculated and also the effect of the monomeric composition on the coherence length has been discussed. The magnetic studies show the paramagnetic and diamagnetic nature of homopolymers and copolymers. The $\mathrm{X}$-ray diffraction pattern indicates that the copolymers are of amorphous nature.
\end{abstract}

Keywords. Polyaniline; poly(aniline-co-o-anisidine); transport properties.

\section{Introduction}

Polyanilines (PA) constitute a large family of polymers which can be synthesized by electrochemical method (Genies et al 1985; Genies and Tsintavis 1986) or by chemical oxidative polymerization of aniline (MacDiarmid et al 1985; MacDiarmid and Epstein 1989; Gupta and Borkar 1990; Gupta and Umare 1992). The chemical synthesis of polyaniline usually involves the oxidative polymerization of aniline in $\mathrm{HCl}$ by $\left(\mathrm{NH}_{4}\right)_{2} \mathrm{~S}_{2} \mathrm{O}_{8}$ to result in emeraldine hydrochloride salt. The emeraldine hydrochloride salt can be converted to emeraldine base by treating with $\mathrm{NH}_{4} \mathrm{OH}$. The emeraldine base corresponds to $50 \%$ oxidized poly(paraphenyleneamineimine) $\left[\left(-\mathrm{C}_{6} \mathrm{H}_{4}-\mathrm{NH}-\right.\right.$ $\left.\left.\mathrm{C}_{6} \mathrm{H}_{4}-\mathrm{NH}\right)_{y}-\left(\mathrm{C}_{6} \mathrm{H}_{4}-\mathrm{N}=\mathrm{C}_{6} \mathrm{H}_{4}=\mathrm{N}-\right)_{1-y}\right]_{x}$ (Neoh et al 1990). When emeraldine base is equilibrated in a large excess of aqueous acid solution, protonation occurs at imine repeat unit to produce emeraldine salt of polyaniline (PA-ES) believed to have the composition (Umare et al 1995; Trivedi 1999)

$$
\left[\left(\mathrm{C}_{6} \mathrm{H}_{4}-\mathrm{NH}-\mathrm{C}_{6} \mathrm{H}_{4}-\mathrm{NH}\right)_{y}-\left(\mathrm{C}_{6} \mathrm{H}_{4}-\stackrel{+}{\mathrm{N}} \mathrm{H}=\mathrm{C}_{6} \mathrm{H}_{4}=\stackrel{+}{\mathrm{N}} \mathrm{H}\right)_{1-y}\right]_{x},
$$

where $\mathrm{A}^{-}$is the anion and consists of equal number of reduced $\left[\mathrm{C}_{6} \mathrm{H}_{4}-\mathrm{NH}-\mathrm{C}_{6} \mathrm{H}_{4}-\mathrm{NH}\right]$ and oxidized $\left[\mathrm{C}_{6} \mathrm{H}_{4}-\right.$ $\left.\mathrm{N}=\mathrm{C}_{6} \mathrm{H}_{4}=\mathrm{N}-\right]$ repeat units. This results in the increased conductivity. However, conductivity is affected by the protonation, level of oxidation, moisture content, poly-

\footnotetext{
*Author for correspondence
}

merization condition, solvent used etc (Gupta and Umare 1992; Umare et al 1996). The electrical conductivity of chemically synthesized poly(o-anisidine) (PoA) has been studied. It is observed that the electrical conductivity is substantially less than that of PA (Gupta et al 1993; Umare et al 1995, 1996) which may be due to steric constraints imposed by methoxy group that could induce additional deformation along the polymer backbone as well as increase the interchain distance. Both factors reduce the mobility of the carriers and as a result lower conductivity is exhibited.

PA represents a class of quasi-1-dimensional disordered conductor which consists of bundles of well coupled chains instead of isolated chains as in conventional quasi-1-dimensional conductors and within these bundles the electron is 3-dimensionally delocalized (Heeger et al 1988; Wang et al 1991). Thus the electron state in PA-ES is 3-dimensional metallic state in the ordered crystalline regions of the polymers separated from one another by amorphous regions and the formation of bundles is attributed to interchain transfer (Kivelson and Heeger 1988). However, in amorphous regions, the interchains transfer rate is suppressed by disorder, so that the electron is still localized in essentially isolated chain while in PoA inter chain coupling and coherence transfer rate are suppressed due to the presence of $-\mathrm{OCH}_{3}$ group which is non-metallic within crystalline regions.

The copolymerization of aniline and o-anisidine has been performed by chemical oxidation (Umare et al 2000). It has been observed that the amount of o-anisidine incorporated in the copolymer chain is more than 
aniline monomer due to its higher reactivity. In the present paper we have studied experimentally the effect of the o-anisidine content, on the optical, transport properties and X-ray diffraction patterns of copolymers of $\mathrm{PA}$ and PoA.

\section{Experimental}

\subsection{Preparation of copolymers}

Poly(aniline-co-o-anisidine) (PA-co-PoA) copolymers were chemically copolymerized (Siyu et al 1997; Borkar et al 2001) from the monomers, aniline and o-anisidine, using ammonium peroxodisulphate as an oxidant in acid aqueous medium. The molar feed ratio of the starting aniline monomer was varied to result in copolymers of different compositions. The copolymerization was carried out in $\mathrm{N}_{2}$ atmosphere at $0-4{ }^{\circ} \mathrm{C}$.

UV-visible spectra of the homopolymers and copolymers were recorded at room temperature in different solvents viz. 1-methyl-2-pyrrolidone (NMP) (32.4), dimethylformamide (DMF) (36.7), dimethylsulphoxide (DMSO) (46.7) in 190-700 nm range using a UV-240 Shimadzu automatic recording double beam spectrophotometer. The magnetic susceptibility of the polymeric samples was determined by Gouy's method (Inoue and Yanagisawa 1974) at room temperature using mercury tetrakis(thiocyanato)cobaltate (II), $\mathrm{Hg}\left[\mathrm{Co}(\mathrm{CNS})_{4}\right]$ as reference material. Magnetic susceptibility value per tworing mole was computed from the change in mass of the sample under applied magnetic field of different strengths between 2300 and $9800 \times 10^{-4} \mathrm{~T}$. Diamagnetic corrections for the compound and counter ion were applied. X-ray diffractograms were recorded on Philips PW-700 automatic X-ray diffractometer using $\mathrm{Cu} \mathrm{K} \alpha$ radiation of wavelength, $\lambda=0 \cdot 154060$ and $0 \cdot 154434 \mathrm{~nm}$. The diffractometer was operated at $35 \mathrm{kV}$ and $20 \mathrm{~mA}$. Electrical conductivity of the polymeric samples was measured as a function of temperature as resistivity by two-probe method. Powdered samples were made into pellets under a pressure of 5 metric tons $\mathrm{cm}^{-2}$. The resistance was measured as a function of temperature using LCR meter.

\section{Results and discussion}

\subsection{Electronic spectra}

The absorption bands of homopolymers and copolymers in NMP, DMF and DMSO are recorded. The corresponding bands are given in table 1 . There are two absorption bands in the electronic spectra of homopolymers and copolymers. The band around 308-330 nm (4.03-3.76 eV) is assigned to $\pi \rightarrow \pi^{*}$ (bandgap) transition (which is related to the extent of conjugation between the adjacent rings in the polymer chain) and the band above $600 \mathrm{~nm}$ (exciton band) is due to inter band charge transfer associated with excitation of benzenoid to quinoid moieties (formation of exciton). These bands change with solvents. The $\pi \rightarrow \pi^{*}$ band of copolymers shows hypsochromic shift with the increase in dielectric constant of the solvent. Ghosh and Kalpagam (1990) tried to explain such shift on the basis of dielectric constant of the solvent. Macroscopic parameters such as the dielectric constant and refractive index cannot always fully explain the solvatochromism, as it is dependent on many other factors like geometry as well as electronic structures of both solute and solvent. However, the exciton band shows a bathochromic shift with an increase in dielectric constant of the solvent in which the UV-visible absorption spectra were taken. In the case of exciton band the absorption is interchain. The excitation leads to formation of molecular exciton (positive charge on the adjacent benzenoid units and negative charge centred on quinoid unit) (Ginder and Epstein 1990). This interchain charge transfer from HOMO to LUMO may lead to the formation of positive and negative polarons (Stafstrom et al 1987).

A polymer in a solvent of high dielectric constant, may exist in 'coil' like conformation (decrease in conjugation) and a less polar solvent provides thermodynamically more stable chain conformation and restricts the polymer to lower energy high planarity state. Such shift may increase the conjugation of the system which yields a lower energy transition, a red shift (Zho et al 1991). The $\pi \rightarrow \pi^{*}$ band in copolymers shifts to the lower wavelength as the percentage of o-anisidine in copolymer increases which may be due to addition of more methoxy groups which twist the torsion angle, which are expected to increase the average bandgap in the conjugated polymer chain.

\subsection{Magnetic susceptibility}

Magnetic susceptibility, $\chi_{\mathrm{m}}$, per two-ring unit mole and the magnetic moment data are recorded in table 2. The data show that both the homopolymers PA and PoA show paramagnetism whereas copolymers show diamagnetic

Table 1. UV-visible absorption bands of copolymers and homopolymers.

\begin{tabular}{lcccccc}
\hline $\begin{array}{l}\text { Polyaniline } \\
\text { derivative } \\
\text { base form }\end{array}$ & \multicolumn{4}{c}{ UV-visible absorption band (nm) } \\
\cline { 2 - 7 } & \multicolumn{2}{c}{ DMSO } & \multicolumn{2}{c}{ DMF } & \multicolumn{2}{c}{ NMP } \\
\hline PA & 314 & 620 & 328 & 618 & 329 & 620 \\
PA-co-PoA10 & 318 & - & 328 & 614 & 328 & 630 \\
PA-co-PoA20 & 320 & 620 & 324 & 610 & 332 & 620 \\
PA-co-PoA40 & 322 & 628 & 322 & 606 & 328 & 620 \\
PA-co-PoA60 & 322 & 624 & 320 & 600 & 324 & 620 \\
PA-co-PoA70 & 322 & 620 & 318 & 600 & 322 & 614 \\
PoA & 316 & 620 & 308 & 604 & 312 & 622 \\
\hline
\end{tabular}


nature. The homopolymers have large magnetic moments (3-4 unpaired electrons or holes per chain unit). This is equal to $0 \cdot 75 / 1 \cdot 0$ electrons/holes per monomer unit in the chain. The change in magnetic susceptibility of the copolymers may be due to change in unpaired spin concentration (polaron/bipolaron) in the chain units. In the study of the spin dynamics of PA it has been proposed that polaron-polaron reaction or polaron ionization to bipolaron (spin-less charge defects) could change the spin concentration in the sample and hence the magnetic susceptibility (Mizoguchi 1990). The polarons in the $\pi$-conjugated systems could interact with each other or with neighbouring chains leading to spin-less charged states or hole states (Jozefowicz et al 1989; Ginder and Epstein 1990). This state may be free or delocalized depending on the Coulomb forces between the interacting moieties. They can also remain localized in the vicinity of charged counterion formed in inter or intramolecular chains.

\section{$3.3 \quad X$-ray diffraction}

X-ray diffractograms of copolymers show broadening of peaks which indicates copolymers are of amorphous nature while PA is crystalline. The $d$-spacing calculated from angular positions of the reflections is given in table 3

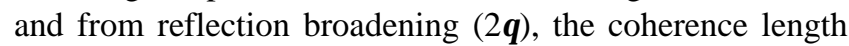
$(L)$ of the crystalline order in the sample is calculated using Scherrer formula (Guinior 1963)

Table 2. Magnetic susceptibility of homopolymers and copolymers.

\begin{tabular}{lcc}
\hline $\begin{array}{l}\text { Magnetic } \\
\text { Polyaniline derivative } \\
\text { hydrochloride salt }\end{array}$ & $\begin{array}{c}\text { Musceptibility, } \chi_{\mathrm{m}} \\
(\mathrm{emu} / 2 \text { ring unit) }\end{array}$ & $\mu_{\text {eff. }}, \mathrm{B.M}$. \\
\hline PA & $6.813 \times 10^{-3}$ & 4.103 \\
PA-co-PoA10 & $-4.345 \times 10^{-3}$ & Diamag. \\
PA-co-PoA20 & $-3.458 \times 10^{-3}$ & Diamag. \\
PA-co-PoA40 & $-2.306 \times 10^{-3}$ & Diamag. \\
PA-co-PoA60 & $-2.908 \times 10^{-3}$ & Diamag. \\
PA-co-PoA70 & $-2.400 \times 10^{-3}$ & Diamag. \\
PoA & $3.781 \times 10^{-3}$ & 3.072 \\
\hline
\end{tabular}

Table 3. Characteristics of X-ray diffraction pattern.

\begin{tabular}{lcc}
\hline $\begin{array}{l}\text { Polyaniline } \\
\text { derivative HCl salt }\end{array}$ & $\begin{array}{c}\text { Coherence length }(\mathrm{L}), \\
(\AA)\end{array}$ & $d$-spacing $(\AA)$ \\
\hline PA & $8 \cdot 15$ & 3.53 \\
PA-co-PoA10 & $8 \cdot 03$ & 3.52 \\
PA-co-PoA20 & $8 \cdot 00$ & 3.54 \\
PA-co-PoA40 & 3.40 & 3.56 \\
PA-co-PoA60 & $4 \cdot 13$ & 3.59 \\
PA-co-PoA70 & 6.90 & 3.63 \\
PoA & 5.90 & 3.59 \\
\hline
\end{tabular}

$$
L=0.9 \lambda / \Delta 2(\theta) \cos \theta .
$$

From table 3 it is observed that there is increase of $d$ spacing and decrease of coherence length as the fraction of o-anisidine increases in the copolymer composition. On increasing the amount of o-anisidine in the copolymer the crystallite size decreases which may be due to the presence of methoxy group on the aromatic ring which increases disorder and decreases crystal size. Thus, in copolymers the increased charge localization may be due to the reduction of interchain diffusion of charge, decrease of interchain band width which is caused by the large transverse unit cell length and decrease in coherence between the chains caused by greater disorder in interchain separation within crystalline region, due to the existence of the side group $\left(-\mathrm{OCH}_{3}\right)$ along the main copolymer chain.

\subsection{Electrical conductivity}

The temperature dependence of conductivity was fitted to an Arrhenius type equation

$$
\sigma_{(T)}=\sigma_{0} \exp (-E a / 2 k T),
$$

and the measured values were plotted semilogarithmically as a function of reciprocal of temperature (figure 1a). The conductivity is found to increase with temperature, however, there are deviations at lower temperatures. The reason for the deviation is not known but the results relate to a small temperature range where Arrhenius behaviour can be regarded as a good approximation to band theory. In the present studies the temperature dependence is fitted to the Zeller equation (Shante et al 1973). Figure 1b describes the interchain conductivity where only the neighbour variable range hopping (VRH) of charge (which is quasi-1-dimensional) is considered

$$
\sigma_{(T)} \alpha \exp \left[-\left(T_{0} / T\right)^{1 / 2}\right]
$$

In Zeller equation, $T_{0}$ is related to delocalization length $\left(\alpha^{-1}\right)$, most probable hopping distance $(R)$ and hopping
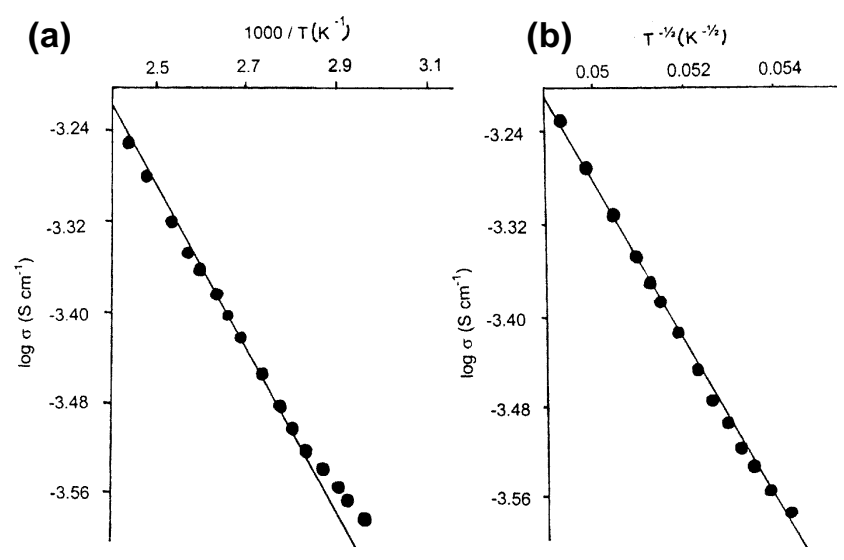

Figure 1. Temperature dependence of electrical conductivity of PA-co-PoA60 (a) arrhenius equation and (b) zeller equation. 
Table 4. Transport properties of copolymers and homopolymers.

\begin{tabular}{lccccc}
\hline $\begin{array}{l}\text { Polyaniline } \\
\text { derivative }\end{array}$ & & & & & \\
$\mathrm{HCl}$ salt & $\sigma(\mathrm{S} / \mathrm{cm})$ & $T_{0}(\mathrm{~K})$ & $\alpha^{-1}(\mathrm{~nm})$ & $R(\mathrm{~nm})$ & $w(\mathrm{eV})$ \\
\hline PA & $6.5 \times 10^{-2}$ & $0.509 \times 10^{4}$ & 4.55 & 4.66 & 0.110 \\
PA-co-PoA10 & $2.7 \times 10^{-2}$ & $1.027 \times 10^{4}$ & 2.26 & 3.29 & 0.221 \\
PA-co-PoA20 & $3.4 \times 10^{-3}$ & $1.197 \times 10^{4}$ & 2.31 & 3.50 & 0.305 \\
PA-co-PoA40 & $1.6 \times 10^{-3}$ & $0.989 \times 10^{4}$ & 2.36 & 3.36 & 0.210 \\
PA-co-PoA60 & $3.4 \times 10^{-4}$ & $1.379 \times 10^{4}$ & 1.97 & 2.83 & 0.297 \\
PA-co-PoA70 & $2.2 \times 10^{-4}$ & $1.673 \times 10^{4}$ & 1.89 & 2.04 & 0.375 \\
PoA & $4.1 \times 10^{-4}$ & $1.973 \times 10^{4}$ & 1.17 & 2.36 & 0.424 \\
\hline
\end{tabular}

energy ( $w$ ) by the relations (4), (5) and (6) (Jozefowicz et al 1989; Pouget et al 1991).

$$
\begin{aligned}
& T_{0}=8 \alpha / N\left(E_{\mathrm{F}}\right) Z k, \\
& R=\left(T_{0} / T\right)^{1 / 2} \cdot \alpha^{-1} / 4, \\
& w=Z k T_{0} / 16,
\end{aligned}
$$

where $Z$ is the number of nearest neighbouring chains $(\sim 4), k$ the Boltzmann constant and $N\left(E_{\mathrm{F}}\right)$ the density of states at fermi energy for sign of spin which is taken as 1.6 states per eV (2-ring unit suggested for PA) (Jozefowicz et al 1989). The value of $T_{0}$ is determined graphically from $\log \sigma_{(T)}$ vs $T^{-1 / 2}$ (figure $1 \mathrm{~b}$ ) and other parameters are computed from the data (table 4$)$. It is observed that $T_{0}$ for PoA is more than PA (maximum localization length). Therefore, correspondingly the localization length and average charge hopping distance decrease on increasing the amount of o-anisidine in copolymer chain. This increase in the electron localization is due to the presence of $-\mathrm{OCH}_{3}$ group in one of the aromatic rings in the copolymer chain which increases the adjacent torsion in the ring angle to avoid strong steric repulsion from the $-\mathrm{OCH}_{3}$ on the ring and $T_{0}$ value increases with more incorporation of o-anisidine in copolymer composition. The temperature dependence of the electrical conductivity fits (3) (figure 1b) for homopolymers and copolymers, which suggests that the charge conduction is quasi-1D VRH between nearest neighbouring chains (Heeger et al 1988; Wang et al 1991). From the values of $T_{0}$ (table 4 ), the localization length is found to decrease and is in the same order as the coherence length (table 3) obtained from the X-ray diffractogram. This shows that the electrons are delocalized within the crystalline regions, the localization length of copolymers is found to be intermediate between those of homopolymers.

\section{Conclusions}

WAXRD studies on homopolymer and copolymer show that they are largely amorphous but the crystalline region belongs to orthorhombic crystalline lattice and the inter- chain spacing and coherence length are affected by the percentage of o-anisidine in copolymer chain. The electrical conductivity behaviours of the copolymers show intermediate characteristics, between those of homopolymer PA and PoA. The values of $T_{0}$ of the copolymers are dependent on the amount of o-anisidine incorporated in copolymer chain. Temperature dependence of electrical conductivity suggests that the copolymers are a class 1-D disorder conductor between the localization state associated by proton exchange as that of homopolymers. Increased electron localization in copolymer can be explained by increased interchain spacing and interchain disorder in copolymer.

\section{Acknowledgements}

Thanks are due to the Director, RSIC, Nagpur University, Nagpur, for recording the XRD pattern. The financial support and the fellowship under FDP (ADB) by UGC, New Delhi, are gratefully acknowledged. One of the authors (SSU) wishes to thank MHRD, New Delhi, for funding the project (No. F. 26-1/98 TS 1).

\section{References}

Borkar A D, Gupta M C and Umare S S 2001 Polym. Plast. Technol. Eng. 40225

Genies E M and Tsintavis C 1986 J. Electro and Chem. 200 127

Genies E M, Syed A A and Tsintavis C 1985 Mol. Cryst. Liq. Cryst. 121181

Ghosh S and Kalpagam V 1990 in Polymer science contemporary themes, (ed.) S Sivaram (New Delhi: Tata MaGraw Hill) Vol. II p. 819

Ginder J M and Epstein A J 1990 Phys. Rev. B41 1510674

Guinior A 1963 X-ray diffraction in crystals, imperfect crystals and amorphous bodies (San Francisco: W H Freeman)

Gupta M C and Borkar A D 1990 Indian J. Chem. A29 631

Gupta M C and Umare S S 1992 Macromolecules 25138

Gupta M C, Warhadpande S V and Umare S S 1993 Indian J. Chem. A32 298

Heeger A J, Kivelson S A, Schrieffer J R and Su W P 1988 Rev. Mod. Phys. 60781 
Inoue H and Yanagisawa S J 1974 Inorg. Nucl. Chem. 361409 Jozefowicz M E, Laversana R, Javadi H S, Epstein A J, Pouget J P, Tang X and MacDiarmid A G 1989 Phys. Rev. B39 12598

Kivelson S A and Heeger A J 1988 Synth. Met. 22371

MacDiarmid A G and Epstein A J 1989 Faraday Discuss. Chem. Soc. 88317

MacDiarmid A G, Chiang J C, Halporn M H, Hvang W S, Mu S L, Somasiri N L, Wu W and Yaniger S I 1985 Mol. Cryst. Liq. Cryst. 121173

Mizoguchi K 1990 Macromol. Chem. Macromol. Symp. 3753

Neoh K G, Kang E T and Tan K L 1990 Eur. Polym. J. 26403

Pouget J P, Jozefowicz M E, Epstein A J, Tang X and MacDiarmid A G 1991 Macromolecules 24779

Shante V K S, Verma C M and Bloch A N 1973 Phys. Rev. 138 4885
Siyu Y, Do N T, Dao L H and Vijh A K 1997 Synth. Met. 65 88

Stafstrom S, Bredas J L, Epstein A J, Woo H S, Taner D B, Huang W S and MacDiarmid A G 1987 Phys. Rev. Lett. 59 1464

Trivedi D C 1999 Bull. Mater. Sci. 22447

Umare S S, Huque M M, Gupta M C and Viswanath S G 1995 Macromol. Rep. A32 281

Umare S S, Huque M M, Gupta M C and Viswanath S G 1996 Macromol. Rep. A33 381

Umare S S, Borkar A D and Gupta M C 2000 Phy. Chem. Chem. Phys. (communicated)

Wang Z H, Li C, Scherr E M, MacDiarmid A G and Epstein A J 1991 Phys. Rev. Lett. 661745

Zho W S, Cromak K and MacDiarmid A G $1991 \mathrm{~J}$. Am. Chem. Soc. 1132665 